\title{
Effective Backoff Scheme to ease the Network Congestion on IEEE 802.11 DCF
}

\author{
BongGi Jun ${ }^{1}$ and Jaehyun $\mathrm{Nam}^{2 *}$ \\ ${ }^{1}$ Silla University, Department of Computer Engineering, \\ ${ }^{2}$ Silla University, Department of Information Technology, \\ Busan, Korea \\ \{bgjun,jhnam\}@silla.ac.kr
}

\begin{abstract}
Since all transmitting stations in the range share the wireless channel, the IEEE 802.11 $M A C$ uses the Binary Exponential Backoff (BEB) algorithm to minimize collision. The BEB technique resets the Contention Window values after each successful frame transmission regardless of the network state, and a sudden $C W$ decrease incurs performance degradation as the number of competing stations increases. To resolve this problem, we propose a new algorithm that adjusts $C W$ values using $(m, k)$-firm scheduling technique. The proposed algorithm changes $C W$ using the violating distance instead of resetting the $C W$ to minimum value after every successful transmission. The performance results are shown to identify the improvement of our scheme in terms of waiting time comparing to the BEB.
\end{abstract}

Keywords: Backoff Algorithm, IEEE 802.11, (m,k)-firm scheme, Contention Window

\section{Introduction}

The purpose of WLANs is to provide communication services with acceptable delay and quality using small portable devices anytime and anywhere. The IEEE 802.11 is the dominant standard for WLANs and includes detailed specifications for both MAC and PHY. The IEEE 802.11 MAC layer protocol incorporates CSMA/CA-based DCF for accessing the wireless channel. In this method, station becomes immediately ready to transmit a frame when an idle period of the wireless channel exceeds the DIFS. Thus, a station with a packet to be transmitted observes the channel activity until the channel is sensed idle during a DIFS period. Since all stations within the range share a single wireless channel, the IEEE 802.11 MAC uses a contention resolution method called the Binary Exponential Backoff (BEB) algorithm to minimize collisions generated by multiple simultaneous transmissions. In this algorithm, a random backoff time is chosen in the interval range [0, CW-1] when the channel is sensed busy. The value of CW is called the contention window.

The IEEE 802.11 MAC does not provide the service of traffic priorities in each station, and the BEB algorithm computes the CW value size according to success/failure of transmitted frames regardless of wireless network conditions. The DCF does not distinguish what kinds of traffic they are transmitting and the channel access probability is the same at all stations statistically. Moreover, the backoff counter at every station depends on the number of collisions. In particular, by resetting the $\mathrm{CW}$ to the initial contention window, $\mathrm{CW}_{\text {min }}$ after successful transmission, a station is likely to experience collisions and retransmissions.

* Corresponding Author 
In this paper, we proposes a simple and effective contention window resetting scheme using $(\mathrm{m}, \mathrm{k})$-firm scheduling technique to reduce potential collisions generated by resetting the $\mathrm{CW}$ to the $\mathrm{CW}_{\min }$. The proposed technique reduces collision probability created from a rapid increase/decrease in $\mathrm{CW}$ by setting the $\mathrm{CW}$ values based on violation distance of $(\mathrm{m}, \mathrm{k})$-firm technique. The performance results are shown to identify the improvement of our scheme in terms of waiting time comparing to the BEB.

The paper is organized as follows. Section 2 contains related works. Section 3 introduces the IEEE 802.11 DCF and presents our proposed backoff algorithm protocol. Sections 4 discuss performance evaluation of the proposed routing protocol against existing routing protocols in simulation. Section 5 concludes the paper.

\section{Related Works}

Several works on slowly decreasing the CW value were proposed [2-7]. [3] and [4] suggested an exponential decrease in the CW value upon a successful transmission instead of resetting to $\mathrm{CW}_{\min }$, but they assumed a fixed scale of decrease without taking the network conditions into ac-count. While [2] and [5] suggested slow decrease backoff algorithms to adapt to the network load, there was another proposal to assume a p-persistent MAC protocol in which the station would transmit with a probability $p$ and refrain from transmitting with a probability $(1-p)[6,7]$. In both the slow decrease and p-persistent cases, complex computations were needed to update the p-value and to estimate the network load, respectively.

Bianchi [8] proposed a simple and accurate analytical model to compute saturation throughput. Wu et al. [9] improved Bianchi's model to consider retry limit. However, these models are not for priorities.

Deng and Chang [10] proposed a priority scheme by differentiating the backoff window: the higher priority class uses the window $\left[0,2_{j+1}-1\right]$ and the lower priority class uses the window $\left[2_{j+1}, 2_{j+2}-1\right]$, where $\mathrm{j}$ is the backoff stage.

Aad and Castelluccia [11] proposed a priority scheme by differentiating interframe spaces (IFS). Pallot and Miller [12] proposed an interesting prioritized backoff time distribution mechanism in which the backoff time is chosen in the current window range with different distributions for different priorities.

\section{The Backoff Algorithm to Easy the Network Congestion}

\subsection{IEEE 802.11 DCF}

The IEEE 802.11 MAC layer protocol provides asynchronous, time-bounded, and contention-free access control. The DCF uses a CSMA/CA technique as the basic MAC mechanism in the 802.11 WLAN. A station with a packet to be transmitted observes the channel activity until the channel is sensed idle during a DIFS period. After the DIFS period, the transmitting station enters the backoff procedure. The backoff time counter is composed of several time slots uniformly and randomly chosen from [0, CW-1]. At the first transmission attempt, the $\mathrm{CW}$ is set to $\mathrm{CW}_{\min }$. The $\mathrm{CW}$ is doubled after each transmission failure and is reset to $\mathrm{CW}_{\min }$ after every successful transmission. In the backoff procedure, the backoff counter would be decremented by 1 in every idle time slot.

When the channel becomes busy again during backoff procedure, the station freezes its own backoff counter. On the other hand, the counter resumes decrementing when the channel becomes idle again for a DIFS time. The station immediately transmits a frame when the backoff counter becomes 0 . 
The DCF collision avoidance procedure uses the BEB strategy. A collision occurs when more than two stations attempt data frame transmission simultaneously after deferring. When the transmitting station detects the collision, a new backoff procedure is performed again with a double-sized CW. In the IEEE 802.11 DCF, the CW is established as follows:

$$
C W_{\text {new }}^{\mathrm{i}}= \begin{cases}\alpha \times C W_{\text {curvent }}^{\mathrm{i}-1}, & \text { transmission failure }- \text { up to max value } \\ C W_{\min } & \text { transmission success }\end{cases}
$$

\subsection{Implementation of the backoff scheme using the (m,k)-firm scheme}

The BEB technique forgets about previous collision experience and resets the $\mathrm{CW}$ after each successful frame transmission regardless of the network conditions. Since the BEB tends to works well with less competing stations, a sudden increase in the number of competing stations generates network performance degradation. Thus, the number of stations attempting to access the channel increases in case of network congestion and failure probability of frame transmission increases. When the $\mathrm{CW}$ is reset to the minimum value after a successful frame transmission, network congestion is worsened.

To resolve this problem, we propose the backoff scheme using the $(\mathrm{m}, \mathrm{k})$-firm scheme (MKBS) to adjust the contention window value. When transmitting data from each station, the MKBS gives high priority to a lot of colliding stations by applying the short backoff time rather than applying the existing backoff algorithm. In [13], (m,k)-firm technique was also used to calculate the $\mathrm{CW}$. However, in [13], CW value was set according to each priority assigned by dividing the maximum $\mathrm{CW}$ value into three ranges. In this technique, network congestion keeps getting worse because the $\mathrm{CW}$ is reset to the $\mathrm{CW}_{\min }$ after successful transmissions. In the MKBS, the gradual $\mathrm{CW}$ decrease was induced using the violating distance of $(\mathrm{m}, \mathrm{k})$-firm instead of resetting to the $\mathrm{CW}_{\min }$ value after successful transmissions.

The $(\mathrm{m}, \mathrm{k})$-firm stream scheme states that $\mathrm{m}$ out of any $\mathrm{k}$ consecutive packets in the stream must meet their respective deadline [14]. A task with $(\mathrm{m}, \mathrm{k})$-firm is in a success state if $\mathrm{m}$ out of its last $\mathrm{k}$ consecutive packets meet the deadline, otherwise it is in a failure state. For each stream source, which requires an $(\mathrm{m}, \mathrm{k})$-firm, the priority is assigned based on the number of consecutive deadline misses that leads the stream to violate its $(\mathrm{m}, \mathrm{k})$-firm requirement. This number of deadline misses is referred to as distance to failure state from current state.

The k-sequence is a word of $\mathrm{k}$ bits ordered from the most recent to the oldest job in which each bit keeps memory of whether the deadline is missed (bit $=0)$ or met (bit=1). Each new job causes a leftward shift of all the bits, the leftmost exists from the word and is no longer considered, while the rightmost will be a 1 if the job has met its deadline or a 0 otherwise.

The priority of its job at a given instant can be assigned with the distance of the current ksequence to a failure state. Evolving the k-sequence can determine the violating distance of the job. That is left shift the k-sequence and adding in the right side 0s until the evolved ksequence violates $(\mathrm{m}, \mathrm{k})$-firm of the job, and the number of added $0 \mathrm{~s}$ is the priority. If a job stream is already in failure state, the highest priority 0 is assigned.

Normally, for a job with constraint $\beta=(\mathrm{m}, \mathrm{k})$-firm, let priority $V D^{\beta}$ denote its violating distance, we get

$$
V D^{\beta}=k-l(m, s)+1
$$

where $s$ denote the state of the previous $k$ sequence jobs, $l(m, s)$ denote the position (from the right) of the $n t h$ meet (or 1) in the $s$. 
The MKBS proposed in this paper uses the violating distance of (m,k)-firm scheme as the parameter, $\alpha$ of the $\mathrm{CW}$ in backoff procedure. Thus, the CW in the MKBS is calculated as follows:

$$
C W_{\text {new }}^{\mathrm{i}}= \begin{cases}2^{V D^{y}} \times C W_{\text {current }}^{\mathrm{i}-1}, & \text {, the number of transmission } \geq k \\ \alpha \times C W_{\text {current }}^{\mathrm{i}-1} & \text {, the number of transmission }<k\end{cases}
$$

where $V D^{\beta}$ represents violating distance and $\beta$ denotes $(\mathrm{m}, \mathrm{k})$-firm constraint.

If the collision occurs at a station and the probability of $(\mathrm{m}, \mathrm{k})$-firm constraint violation increases, $V D^{\beta}$ is gradually decreased. In contrast, $V D^{\beta}$ gradually increases when the probability of constraint violation is lowered with consecutive successful transmissions. Therefore, the problem of network congestion is resolved by reducing a sudden decrease of $\mathrm{CW}$ to the $\mathrm{CW}_{\text {min }}$ after successful transmissions in the IEEE 802.11 DCF.

When the CW is calculated, the backoff time is determined as follows:

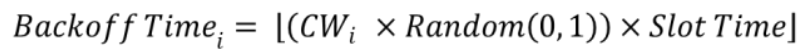

\section{Performance Evaluation}

We implemented the MKBS in Python 2.6 with SimPy-2.1.0. We consider the general case where a set of nodes is scattered in an area such that not all nodes are within transmission range of each other. Table 1 describes the detailed setup for our simulator.

\section{Table 1. Parameters}

\begin{tabular}{|c|c|}
\hline Packet payload & 8184 bits \\
\hline MAC header & 272 bits \\
\hline PHY header & 128 bits \\
\hline Channel Bit Rate & $1 \mathrm{Mbps}$ \\
\hline Propagation Delay & $1 \mathrm{us}$ \\
\hline Slot Time & $50 \mathrm{us}$ \\
\hline SIFS & $28 \mathrm{us}$ \\
\hline DIFS & $128 \mathrm{us}$ \\
\hline
\end{tabular}

Figure 1 shows the comparison of mean waiting time before frame transmission in each station. As shown in the figure, the waiting time in the MKBS was shorter than that of the $\mathrm{BEB}$ as the number of stations increased. The outcome is because the $\mathrm{CW}$ is regulated according to violating distances, instead the station increases its $\mathrm{CW}$ exponentially in the case of transmission collision. 


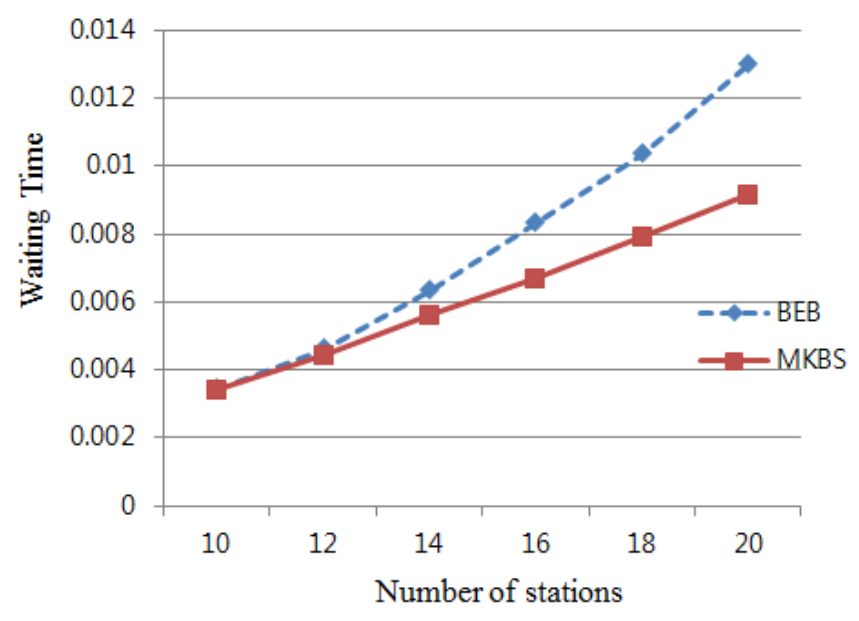

Figure 1. Waiting time vs. number of stations

\section{Conclusion}

We propose the MKBS by modifying the existing BEB algorithm to improve performance of the IEEE 802.11 DCF. The MKBS scheme is mainly characterized by consideration of network congestion conditions by introducing the notion of $(\mathrm{m}, \mathrm{k})$-firm scheme in calculating the $\mathrm{CW}$ value. In the existing $\mathrm{BEB}$ algorithm, it resets the $\mathrm{CW}$ value to the $\mathrm{CW}_{\text {min }}$ after every successful transmission regardless of network conditions, and the station transmits its frame prior to other stations in next transmission. Consequently, the network congestion is aggravated. To resolve this problem, the MKBS minimized a sudden change in the $\mathrm{CW}$ value by controlling the value using the violating distance. The simulation results have shown significant improvement in IEEE 802.11 performance measures upon using MKBS over BEB.

\section{References}

[1] IEEE, 802.11 WG, Part 11: Wireless LAN Medium Access Control (MAC) and Physical Layer (PHY) Specification, (1999).

[2] Q. Ni, I. Aad, C. Barakat and T.Turletti, "Modeling and analysis of slow CW decrease for the IEEE 802.11 MAC protocol”, Proceeding of PIMRC 2003, Beiging, China, (2003) September.

[3] P. Chatzimisios, A. C. Boucouvalas, V. Vitsas, A. Vafiadis, A. Economidis and P. Huang, "A Simple and Effective backoff scheme for the IEEE 802.11 MAC Protocol", Proceedings of CITSA 2005, Orlando, FL, USA, (2005) July.

[4] J. A. Moura and R. N. Marinheiro, "Mac approaches for QoS enhancement in wireless LANs", Proceedings of JETC 2005, Lisbon, Portugal, (2005) November.

[5] B. Li and R. Battiti, "Achieving optimal performance in IEEE 802.11 wireless LANs with the combination of link adaptation and adaptive back off", Computer Networks Journal, Elsevier Science BV, vol. 51, no. 6, (2007), pp. 1574-1600.

[6] F. Cali, M. Conti and E. Gregori, "IEE 802.11 protocol:Design and performance evaluation of an adaptive backoff mechanism”, IEEE Journal on Selected Areas in Communications, vol. 18, no. 9, (2000), pp. 17741786.

[7] R. Brumo, M. Conti and E. Gregori, "A simple protocol for the dynamic tuning of the backoff mechanism in IEEE 802.11 networks", Computer Networks Journal, Elsevier Science BV, vol. 37, no. 1, (2001), pp. 33-44.

[8] G. Bianchi, "Performance analysis of the IEEE 802.11 distributed coordination function", IEEE, J. Sel. Areas Communications, vol. 18, no. 3, (2000) March, pp. 535-547. 
[9] H. Wu, Y. Peng, K. Long, S. Cheng and J. Ma, "Performance of reliable transport protocol over IEEE 802.11 Wireless LANs: Analysis and enhancement", in IEEE Information Communications (INFOCOM), New York, (2002), pp. 599-607.

[10] D. -J. Deng and R. -S. Chang, "A priority scheme for IEEE 802.11 DCF access method", IEICE, Trans. Commun., vol. E82-B, no. 1, (1999) January, pp. 96-102.

[11] I. Aad and C. Castelluccia, "Differentiation mechanisms for IEEE 802.11", in IEEE Information Communications (INFOCOM), Anchorage, AK, (2001), pp. 209-218.

[12] X. Pallot and L. E. Miller, "Implementing message priority policies over an 802.11 based mobile ad hoc network", in IEEE Military Communications Conf. (MILCOM), McLean, VA, ( 2001), pp. 860-864.

[13] B. G. Jun and J. H. Nam, "Modified Backoff Algorithm considering Priority in IEEE 802.11", International Workshop Database, Networking and Communication 2013, Jeju, Korea, (2013) December, pp. 32-35.

[14] M. Hamdaoui and P. Ramanathan, "A Dynamic Priority Assignment Technique for Streams with (m,k)-Firm Deadlines", IEEE, Transactions on Computers, (1994).

\section{Authors}

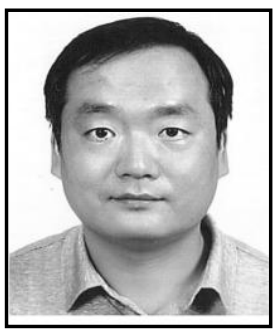

\section{Bonggi Jun}

He received the B.S. degree, M.S. degree and Ph.D. degree from Department of Computer Engineering, Pusan national University, Republic of Korea in 1991, 1993 and 2003 respectively. Since September 2003, he has been as associate professor at Silla University, Republic of Korea. His research interests are in ubiquitous sensor networks and spatio-temporal databases.

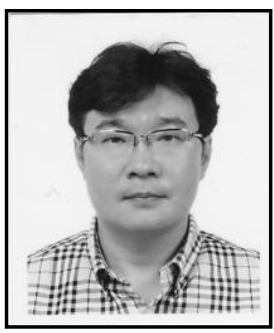

\section{Jaehyun Nam}

He received the B.S. degree, M.S. degree and Ph.D. degree from Department of Computer Engineering, Pusan national University, Republic of Korea in 1989, 1992 and 2002 respectively. Since November 2002, he has been as associate professor at Silla University, Republic of Korea. His research interests are in ubiquitous sensor networks. 